\title{
Interactive comment on "Outer Van Allen belt trapped and precipitating electron flux responses to two interplanetary magnetic clouds of opposite polarity" by Harriet George et al.
}

\section{Harriet George et al.}

harriet.george@helsinki.fi

Received and published: 23 June 2020

General referee comment: This manuscript compares two events in which ICMEs impact Earth's magnetosphere and cause responses in both trapped and precipitating radiation belt electrons. They qualitatively compare and contrast the timing, characteristics, and magnetospheric response during these two events, one of which has magnetic cloud orientation and rotation of Bz North to South, and the other South to North. They describe in detail both events, but as currently presented, it is difficult to directly compare the events or attribute their differences specifically to the ICME characteristics. I recommend the following specific comments be addressed to help clarify 
the manuscript and its findings.

Response: We thank the referee for the careful reading of our manuscript and their constructive comments. We have revised the paper accordingly. Please find our responses below.

Referee comment: It is difficult to compare and contrast the two events directly as they are currently presented. Please consider incorporating some of the following potential suggestions to help the reader better identify the key take-aways from the two-event comparison: - Combining Fig 1 and 3,2 and 4 (as left and right panels, e.g.) would help the reader better look at the relative timing and magnitude of the magnetospheric response during these two events

Response: Figures 1 and 3 , and 2 and 4 have been combined as suggested. These figures are attached below

Referee comment: Including a summary table or figure in the discussion of the different key parameters that were investigated and their similarities/differences between events would also help, since there is a lot of detailed description and text in the discussion section to sift through.

Response: A color-coded summary table of selected solar wind conditions, wave activity, and electron flux response during the sheath, ejecta, and recovery phases of the two events has been added. This table is on page 22 of the revised manuscript.

Referee comment: Is there a reason the EMIC wave observations are taken from GOES rather than Van Allen Probes? Please include the MLT of both spacecraft during these observations, since this can have a large influence on which wave populations will be sampled.

Printer-friendly version

Response: MLT information for the Van Allen Probes have been added to the Wave Activity figure (attached) to show when they are on the dayside and nightside of the Earth. In this figure, a dashed line represents that the satellite is on the nightside while

Interactive

comment 
a solid line represents dayside satellite location. The following text has been added to the Data and Methods section (line 160 of the revised manuscript) to explain the use of GOES data for the EMIC waves: "RBSP data can be used to calculate local ULF and EMIC wave powers on shorter timescales, but these data are not ideal for the analysis of ULF or EMIC waves over the course of an event. The RBSP travel rapidly over a highly elliptical orbit, so sample a range of plasma environments from different regions of the inner magnetosphere over the course of a half orbit. The ULF waves are global and EMIC waves are long lasting, meaning that they are poorly observed over long time periods by the RBSP. By comparison, the GOES satellites are better suited to ULF and EMIC observations over a longer time period due to their fixed orbit and longer period."

Referee comment: Additionally, summing the hiss and chorus wave power from the Van Allen Probes (as you've done for GOES Pc5 and EMIC measurements) in Fig 2 and 4 would make these plots easier to more quantitatively compare between the two events.

Response: The hiss and chorus wave power has been summed as suggested. This can be seen in the attached Wave Conditions figure.

Referee comment: The plasmapause location might be more useful to show in the figures of radiation belt fluxes $(5,7)$ rather than solar wind data, so that the trapped fluxes inside and outside of the plasmasphere can be better identified in Van Allen Probes data.

Response: We have plotted an overlay of the magnetopause and plasmapause position on the colour maps of precipitating and trapped electron flux, in addition to showing the magnetopause and plasmapause location in the Solar Wind Conditions figure. The overlay of the magnetopause is orange and the plasmapause overlay is magenta. The colour maps with these overlays have been attached.

Referee comment: Was using the P6 channel considered, for comparison to the 
Response: No. Channel P6 measures protons but has significant contamination from relativistic electrons. Measurements from this channel have been previously used in qualitative analysis of relativistic electron precipitation (Peck et. al, $2015,10.1002 / 2014 J A 020817)$ by cross-referencing the P6 measurements with other proton measurements to isolate the electron contamination (Rodger et al., 2010, 10.1029/2008JA014023). However, the P6 channel is not very sensitive and the precipitating fluxes of relativistic electrons is generally quite low due to the low proportion of relativistic electrons in the outer radiation belt to electrons with tens or hundreds of $\mathrm{keV}$. This means that the P6 channel does not always provide reliable data for precipitating electrons (Yando et. al., 2011, 10.1029/2011JA016671), and can therefore be difficult to interpret for use in electron precipitation studies. As a result of these complications, we elected to focus on direct measurements of electron flux in the lower energy channels rather than attempt to incorporate indirect measurements of high energy electrons.

Referee comment: line 501-503: Why is the precipitation enhancement here (as opposed to at other times) assumed to be due to a trapped flux enhancement? Some further justification of this is needed.

Response: Upon further consideration, we have changed our explanation of this precipitation enhancement. This text: "The observed precipitation flux enhancement occurring prior to the trapped flux enhancement is likely not due to a true increase in precipitating flux at this time, but rather due to the $90^{\circ}$ POES telescope measuring the trapped flux enhancement before it was measured by RBSP." ... has been changed to: "The precipitating flux enhancement preceding the trapped flux enhancement may be due to increased chorus and EMIC wave activity at this time causing greater scattering of the existing lower energy populations into the bounce-loss cone. A different mechanism may have then caused the trapped flux enhancement at a slightly later time."
ANGEOD

Interactive

comment
Printer-friendly version

Discussion paper 
Referee comment: This also raises the general question of how to interpret the POES data as it is presented, if enhancements can be due just as easily to enhancements in trapped fluxes as enhancements in precipitation. While the caveats of the current technique for presenting the POES data (equation 1 of the manuscript) are nicely mentioned, it is not clear how much better they are than presenting just the 0deg telescope measurements for the purposes of this event comparison.

ANGEOD

Response: The following text has been added to the Data and Methods section: "This means that the POES $0^{\circ}$ detectors do not resolve fluxes near the edge of the loss cone in the case of partially filled loss cones. Therefore, the $0^{\circ}$ detector chronically underestimates fluxes in the loss cone... Despite these drawbacks, we expect this approach for precipitating flux will be superior to the direct $0^{\circ}$ telescope measurements, because it attempts to provide a more accurate estimate of the loss cone fluxes for the latitudes evaluated in this study."

Technical Corrections: 1. please double check the color bar axis units (e.g. Fig 5) - it looks like the REPT $>3.4 \mathrm{MeV}$ fluxes are larger than those in MagEIS $>346$ and $>1079$ $\mathrm{keV}$ channels. This has been corrected. REPT units were per MeV while MagEIS were per keV. The data has been adjusted so all fluxes are per keV 2. line 381 - typo: "decreases" should be "decreased" or "decreasing" Corrected: decreases -> decreasing 3. line 387 - depletion at "high L-shell" is discussed, but then referenced as "low latitude" - shouldn't higher L shells map to higher latitudes? Corrected: low latitude -> high latitude 4. line 400 - typo: "event 2 exhibits moderate level" -> "moderate levels" Corrected: level -> levels

Interactive comment on Ann. Geophys. Discuss., https://doi.org/10.5194/angeo-2020-18, 2020.

Interactive

comment

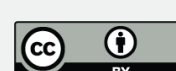




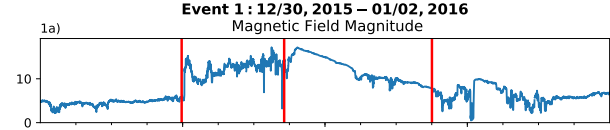

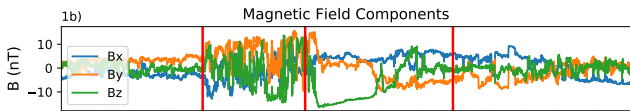
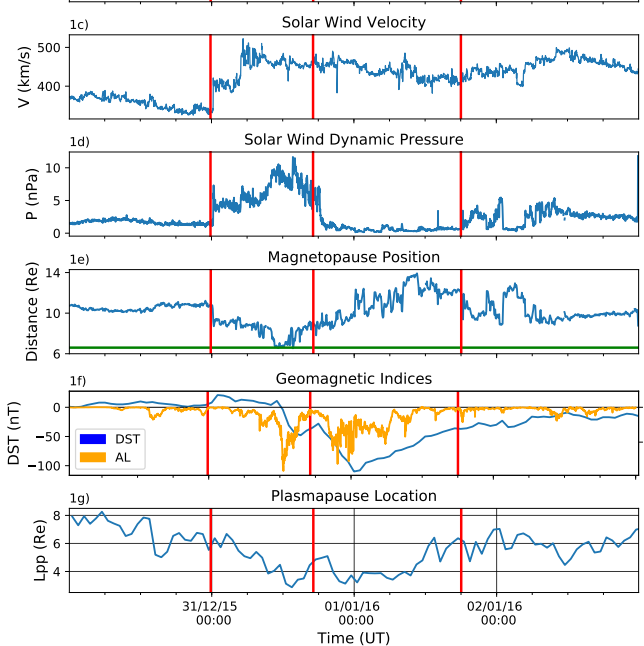

Fig. 1.
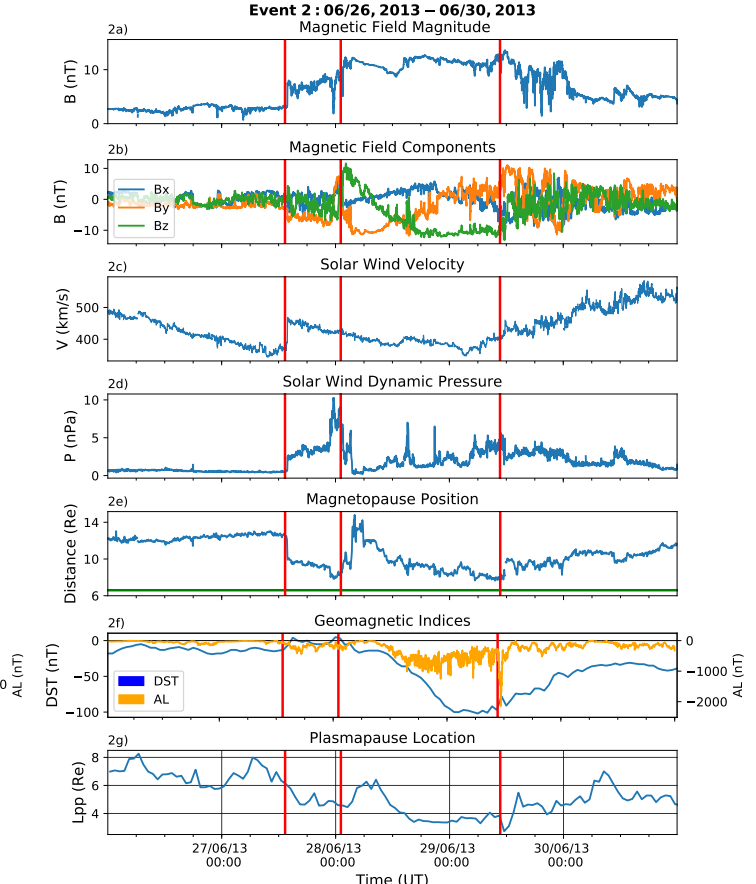

Printer-friendly version

Discussion paper

(c) (i)
Interactive

comment 
ANGEOD

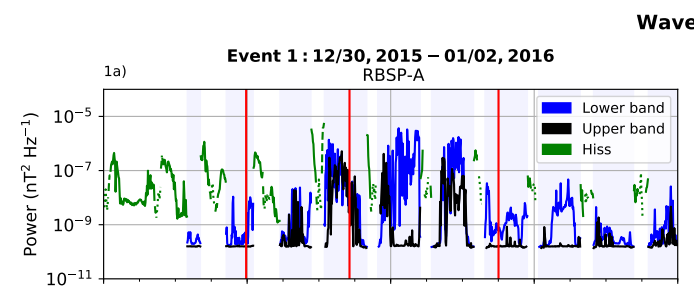

\section{onditions}

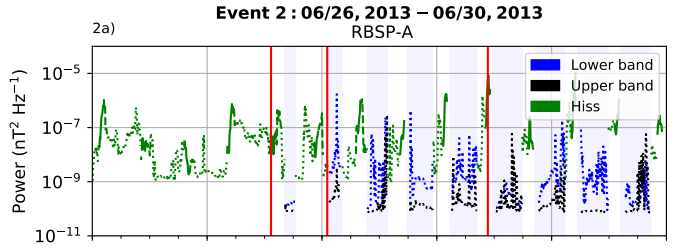

Interactive

comment
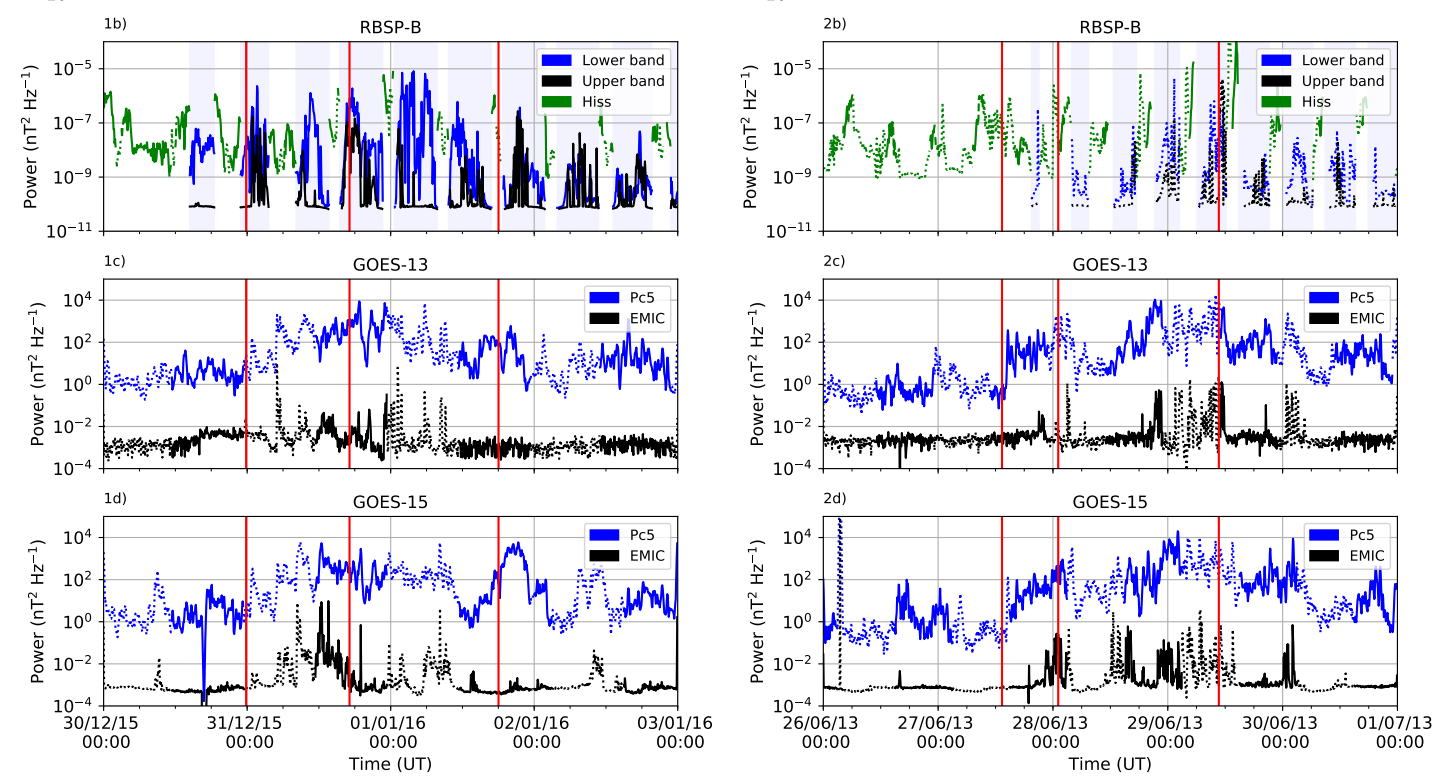

Fig. 2. 

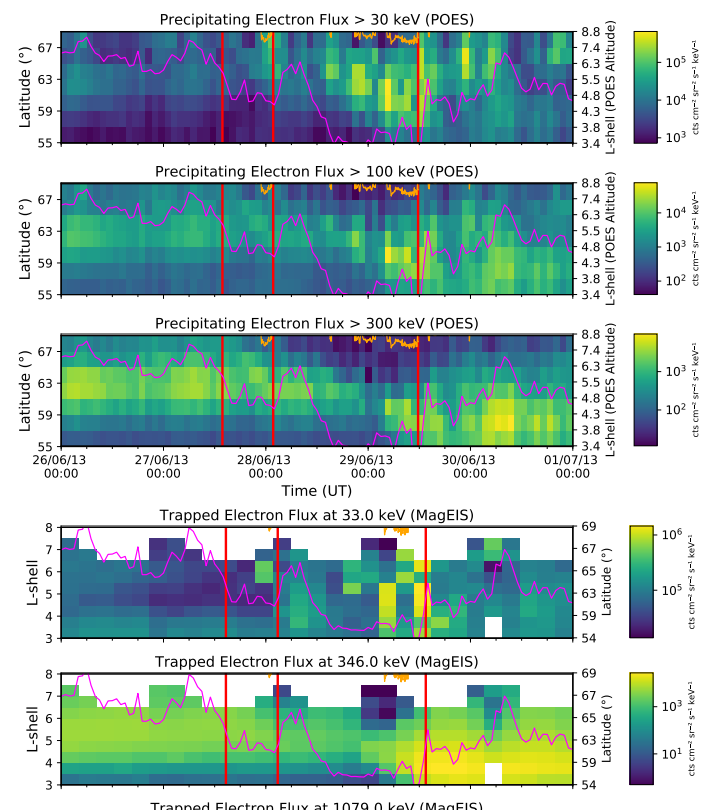

Trapped Electron Flux at $1079.0 \mathrm{keV}$ (MagEIS)
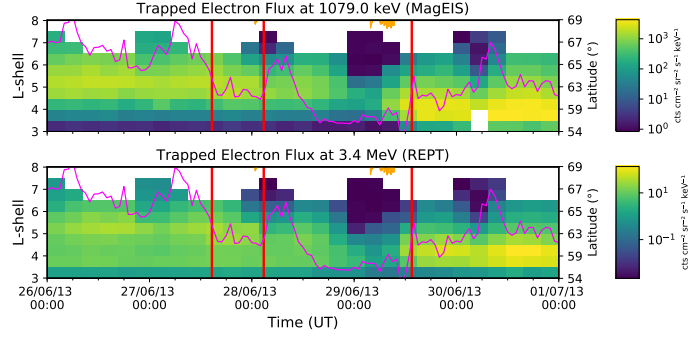

Interactive

comment

Fig. 3. 
Electron Precipitation and Flux 30 Dec, 2015 - 02 Jan, 2016
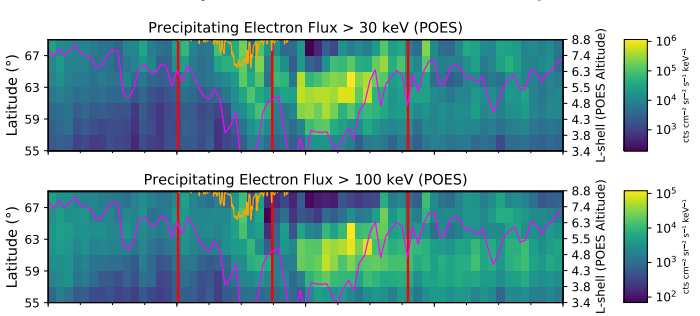

Precipitating Electron Flux $>300 \mathrm{keV}$ (POES)

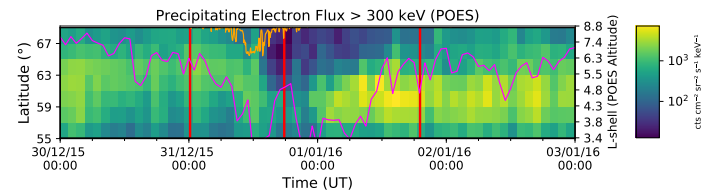

Trapped Electron Flux at $33.0 \mathrm{keV}$ (MagEIS)
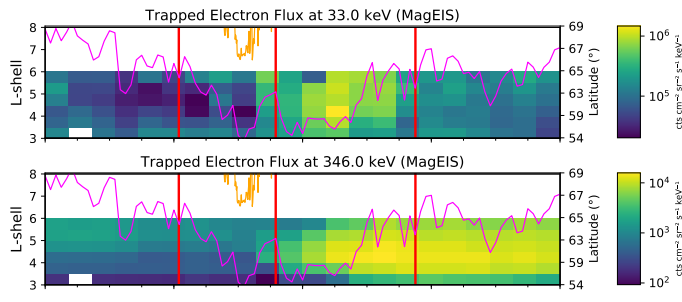

Trapped Electron Flux at $1079.0 \mathrm{keV}$ (MagEIS)

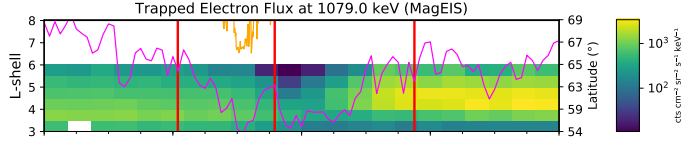

Trapped Electron flux at $3.4 \mathrm{MeV}$ (REPT)

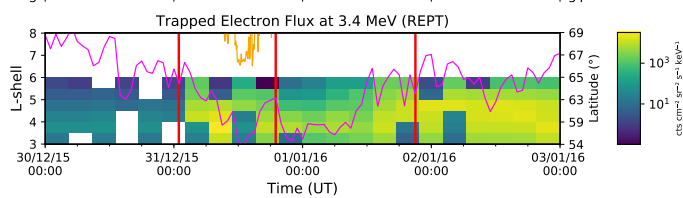

Fig. 4.
ANGEOD

Interactive

comment

Printer-friendly version

Discussion paper

(c) (i) 